\title{
Coulomb and Nuclear Breakup at Low Energies: Scaling Laws
}

\author{
M S Hussein ${ }^{1, a}$, P R S Gomes ${ }^{2, b}$, J Lubian ${ }^{2, c}$, and L F Canto ${ }^{3, d}$ \\ ${ }^{1}$ Instituto de Estudos Avançados and Instituto de Física, Universidade de São Paulo, CP 66318, cep 05314-970, São Paulo, S.P., \\ Brazil \\ ${ }^{2}$ Instituto de Fisica, Universidade Federal Fluminense, Av. Litoranea s/n, Niteroe, R.J. 24210-340, Brazil \\ ${ }^{3}$ Instituto de Fisica, Universidade Federal do Rio de Janeiro, CP 68528, Rio de Janeiro, Brazil
}

\begin{abstract}
We report on a recent work on the low-energy behavior of the breakup cross section in so far as it has important role in the fusion of weakly bound and halo nuclei at near-barrier energies. We assess the way the nuclear component of this cross section scales with the target mass. In complete accord with previous finding at higher energies we verify that the low energy behavior of the breakup cross section for a given projectile and relative center of mass energy with respect to the Coulomb barrier height scales as the cubic root of the mass number of the target. Surprisingly we find that the Coulomb component of the breakup cross section at these low energies also obeys scaling, but with a linear dependence on the target charge. Our findings are important when planning for experiments involving these exotic nuclei.
\end{abstract}

The effect of the coupling to the breakup channel on the complete fusion of weakly bound, and especially, halo nuclei, has been under great scrutiny both experimentally and theoretically [1]. It seems that this coupling hinders the fusion at energies above the barrier, and enhances the tunneling-dominated fusion below the barrier [2]. Owing to the importance of the breakup channel at these low energies, we make an effort to understand its cross section, and the relative importance of its Coulomb and the nuclear components. In particular, we investigate the dependence of these components on the mass number of the target nucleus. To be more specific we mention that in analyzing data on elastic breakup, the cross section is written as the sum of the Coulomb breakup piece, plus the nuclear breakup piece, plus the interference term.

$$
\sigma^{b u}=\sigma_{C}^{b u}+\sigma_{N}^{b u}+\sigma_{\text {interference }}^{b u}
$$

The interest lies in $\sigma_{C}^{b u}$, as through it one can extract information about the collective response of halo and other weakly (bound) nuclei. What is first done (wrongly) is to ignore $\sigma_{\text {interference }}^{b u}$, and consider $\sigma_{N}^{b u}$ to scale linearly with the mass number of the target nucleus. We rely on a recent study of this topic, especially with regards to the dependence on the target mass, made at higher energies, $[3,4]$. According to this study, the nuclear breakup cross section behaves at a given value of the bombarding energy, $E_{\text {Lab }}$ as,

$$
\sigma_{N}^{b u}=P_{1}+P_{2} A_{T}^{1 / 3} .
$$

\footnotetext{
ae-mail: hussein@if.usp.br

be-mail: paulogom@if.uff.br

ce-mail: lubian@if.uff.br

de-mail: canto@if.ufrj.br
}

In the above $P_{1}$ and $P_{2}$, given in $\mathrm{mb}$, are functions of the bombarding energy, and structure of the projectile. This formula, well substantiated by extensive continuum discretized coupled channels calculation in [3], is used to estimate the nuclear cross section for breakup on a heavy target, given the experimental value of the cross section on a light target such as ${ }^{12} \mathrm{C}$, where the Coulomb breakup is usually much smaller. We mention here that [5] also derive a scaling law, but finds a different dependence on the target mass. For the heavy target case, the interest resides in exactly the Coulomb breakup, through which the dipole response can be extracted. In the procedure the interference between the nuclear and Coulomb contributions is ignored which is not always justified [3]. As mentioned above, the next step used by practitioners is to measure the breakup of, say, ${ }^{11} \mathrm{Be}$ on a ${ }^{12} \mathrm{C}$ target and assume it is predominantly nuclear, then extrapolate to the heavy targets such as the case of nuclear breakup contribution to ${ }^{11} \mathrm{Be}$ $+{ }^{208} \mathrm{~Pb}$ [8? ] Once $\sigma_{N}^{b u}$ is calculated then it is subtracted from $\sigma^{b u}$ to extract $\sigma_{C}^{b u}$, and from it the dipole response,

$$
\sigma_{C}^{b u}=\left(16 \pi^{3} / 9\right) \alpha \int d E_{x} n_{E 1}\left(E_{x}\right) \frac{d B(E 1)}{e^{2} d E_{x}}
$$

where $n_{E 1}$ is the number of virtual photons, $\alpha$ is the fine structure constant, and $d B(E 1) / e^{2} d E_{x}$ is the dipole response. Of course the above procedure is faulty as the interference term could be important, as demonstrated in $[3,6]$.

In Fig. 1 we show the results of the CDCC calculation of [3] for the nuclear breakup cross sections of ${ }^{8} \mathrm{~B}$, ${ }^{11} \mathrm{Be}$, and ${ }^{7} \mathrm{Be}$, as functions of the mass number, at different bombarding energies. The non-halo projectile, ${ }^{7} \mathrm{Be}$, seems to obey the scaling law quite well. The halo nu- 


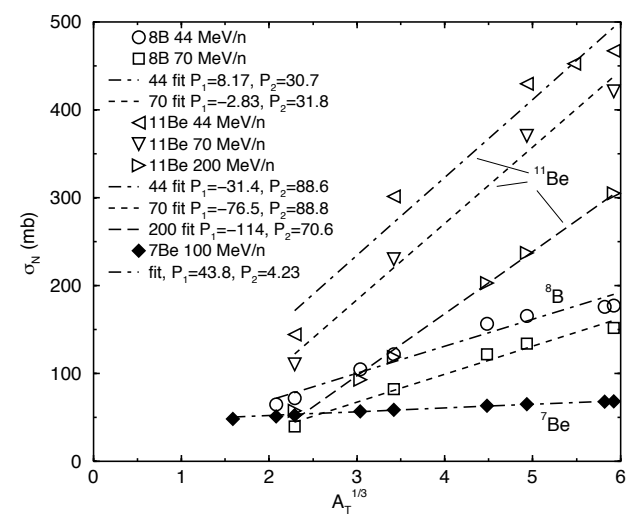

Figure 1. The points show the result of CDCC calculations of the elastic nuclear breakup cross-section for ${ }^{8} \mathrm{~B},{ }^{11} \mathrm{Be}$ and ${ }^{7} \mathrm{Be}$ projectiles at the indicated energies, as a function of target mass number $A_{T}$, along with linear fits. The units of $P_{1}$ and $P_{2}$ are millibarns [3].

clei require values and signs of $P_{1}$ and $P_{2}$ which are not consistent with the simple scaling law, as at high energies these numbers must be positive (see discussion below).

At low energies, the above formula for the cross section is still valid, except that the reference to the energy is made in the form $E / V_{B}$, where $V_{B}$ is the height of the Coulomb barrier, [6]. In the following we derive low energy formula of Eq. (2). We start with the Wong formula, [9], for the fusion cross section taken to be the total reaction cross section without breakup. We write the Wong formula in a slightly different form

$$
\sigma_{F}=\frac{\Gamma}{E} \pi R^{2} \ln \left[1+\exp \left(\frac{1}{\Gamma} \frac{\hbar^{2} \Lambda_{C}^{2}}{2 \mu R^{2}}\right)\right]
$$

where $\Gamma$ is an energy width related to the curvature of the Coulomb barrier as $\Gamma=\hbar \omega / 2 \pi$, and $\mathrm{R}$ is position of the top of this barrier. $\Lambda_{C}$ is the critical angular momentum associated with fusion, $\hbar^{2} \Lambda_{C}^{2}=2 \mu R^{2}\left[E-V_{B}\right]$, where $V_{B}$ is the height of the Coulomb barrier and $R$ is its position.

The total reaction cross section can be written as,

$$
\sigma_{R}=\frac{\Gamma}{E} \pi R^{2} \ln \left[1+\exp \left(\frac{1}{\Gamma} \frac{\hbar^{2}\left(\Lambda_{C}+\Delta\right)^{2}}{2 \mu R^{2}}\right)\right]
$$

where $\Delta$ is the increase in the critical angular momentum arising from the presence of other processes besides fusion. We consider in the following only breakup as contributing to these peripheral reactions. Accordingly $\Delta$ measures the contribution of breakup. Thus the nuclear breakup cross section is taken to be related to the difference, $\sigma_{R}-\sigma_{F}$,

$$
\sigma_{N}^{b u}=\sigma_{R}-\sigma_{F}=\frac{\Gamma}{E} \pi R^{2} \ln \left[\frac{1+\exp \left(\frac{1}{\Gamma} \frac{\hbar^{2}\left(\Lambda_{C}+\Delta\right)^{2}}{2 \mu R^{2}}\right)}{1+\exp \left(\frac{1}{\Gamma} \frac{\hbar^{2} \Lambda_{C}^{2}}{2 \mu R^{2}}\right)}\right]
$$

which can be simplified by expanding to lowest order in $\Delta / \Lambda_{C}$, to give,

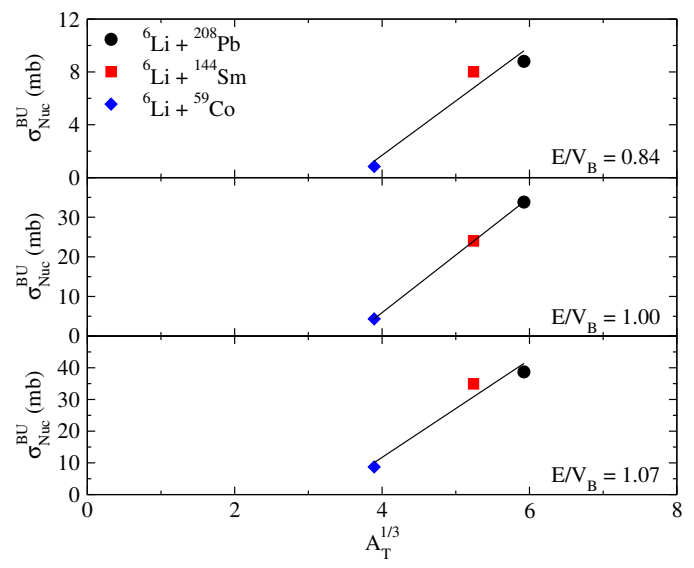

Figure 2. Scaling of the nuclear breakup cross sections as a function of $A_{T}^{1 / 3}$ for the systems discussed in the text, for three energies close to the Coulomb barrier, [6].

$$
\sigma_{N}^{b u}=2 \frac{\pi}{k^{2}} \Lambda_{c} \Delta=2 \pi a\left[1-\frac{V_{B}}{E}\right]\left(R_{P}+R_{T}\right)=P_{1}+P_{2} A_{T}^{1 / 3}
$$

where we have used $\Lambda_{C}^{2}=\left(2 \mu / \hbar^{2}\right)\left(R_{P}+R_{T}\right)^{2}\left[E-V_{B}\right]=$ $k^{2}\left[1-V_{B} / E\right]\left(R_{P}+R_{T}\right)^{2}$, and $\Delta^{2}=k^{2}\left[1-V_{B} / E\right] a^{2}$, with $a$ being the diffuseness of the nuclear surface. Clearly $P_{1}=$ $2 \pi a\left(1-V_{B} / E\right) R_{P}$ and $P_{2}=2 \pi a r_{0}\left(1-V_{B} / E\right)$.

The equation above for $\sigma_{N}^{b u}$ clearly shows that for a fixed $E / V_{B}$ and for a given projectile, the cross section scales linearly with the radius of the target. This was verified recently [6], through a CDCC calculation, shown in Fig.2 below.

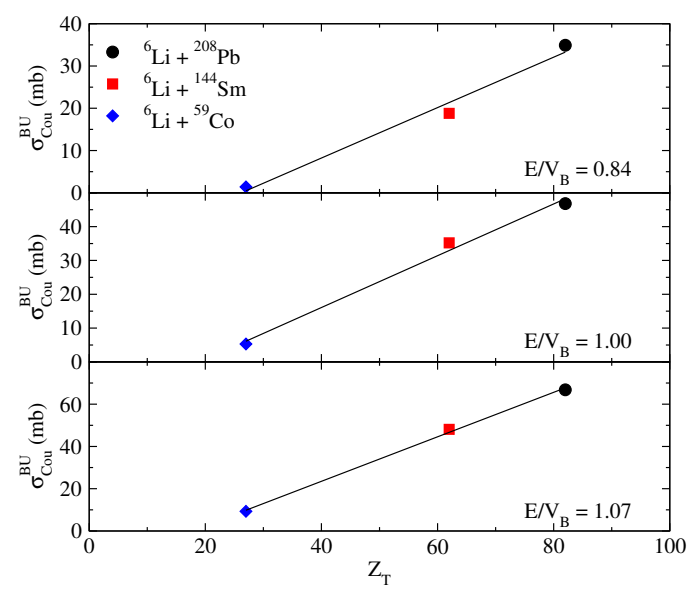

Figure 3. Scaling of the Coulomb breakup cross sections as a function of $Z_{T}$ for the systems discussed in the text, for three energies close to the Coulomb barrier, [6].

The above formula for the nuclear breakup cross section represents the scaling low at low energies and reduces to the one discussed in [3] at higher energies. In [6], the low energy scaling law was confirmed by a detailed CDCC calculation in connection with the effect of breakup on near-barrier energies. The CDCC results for ${ }^{6} \mathrm{Li}$ on three target nuclei shown in Fig. 2 give values of 
$P_{1}$ and $P_{2}$ which are: $\left(E_{c m} / V_{B}=0.84 ; P_{1}=-14.76 m b\right.$, $\left.P_{2}=4.11 \mathrm{mb}\right),\left(E_{c m} / V_{B}=1.00 ; P_{1}=-62.60 \mathrm{mb}\right.$, $\left.P_{2}=16.94 m b\right),\left(E_{c m} / V_{B}=1.07 ; P_{1}=-49.89 m b\right.$, $\left.P_{2}=15.41 \mathrm{mb}\right)$. Only the last case, namely above barrier energy, is qualitatively consistent with the scaling formula. Nevertheless, one can still use this formula at lower energies as the CDCC calculation show. We mention here that the the case $E_{c} m / V_{B}=0.84$ is to be considered with care, as the factor $\left[1-V_{B} / E_{c m}\right]$ is negative here and the scaling law would not be so evident. However, the CDCC calculations performed here seem to show that even at subbarrier energies $\sigma_{b u}$ does depend on the target mass approximately as $A_{T}^{1 / 3}$, and accordingly follow the scaling law. This result may be traced to barrier tunneling effects in $\sigma_{b u}$.

The Coulomb breakup cross sections calculated in [6] also exhibit scaling when plotted as functions of the target charge. Fig. 3 shows the results. This scaling can be understood from the low energy behavior of the Coulomb dissociation cross section [10].

The above discussion about the scaling of the nuclear and Coulomb breakup cross sections at near-barrier energies, should be useful in the study of low energy fusion of weakly bound nuclei as it provides means to assess the feasibility of performing a given experiment which aims to discern the influence of breakup on fusion. More details concerning the material presented here can be found in $[3,6]$. It should be mentioned that the low energy scaling law for the nuclear breakup cross section is of practical use in the planning of experiments to study the influence of breakup on fusion of weakly bound nuclei at near-barrier energies.

\section{Acknowledgements}

The authors would like to thank the financial support from CNPq, CAPES, FAPERJ, FAPESP and the PRONEX.

\section{References}

[1] L. F. Canto, P. R. S. Gomes, R. Donangelo, and M. S. Hussein, Phys. Rep. 424, 1 (2006).

[2] M. Dasgupta, P. R. S. Gomes, D. J. Hinde, S. B. Moraes, R. M. Anjos, A. C. Berriman, R. D. Butt, N. Carlin, J. Lubian, C. R. Morton, J. O. Newton, and A. Szanto de Toledo, Phys. Rev. C 70, 024606 (2004).

[3] M. S. Hussein, R. Lichtenthaler, F. M. Nunes, and I. J. Thompson, Phys. Lett. B 640, 91 (2006).

[4] J. C. Aquadro, D. Pereira, M. S. Hussein, and O. Sala, Phys. Lett. B 100, 381 (1981).

[5] M. A. Nagarajan, C. H. Dasso, S. M. Lenzi, and A. Vitturi, Phys. Lett. B 503, 65 (2001).

[6] D. R. Otomar, P. R. S. Gomes, J. Lubian, L. F. Canto, and M. S. Hussein, Phys. Rev. C 87, 014615 (2013).

[7] T. Nakamura et al., Phys. Rev. Lett 83, 1112 (1999).

[8] T. Aumann et al., Phys. Rev. C 59, 1252 (1999).

[9] C. Y. Wong, Phy. Rev. C 86, 064603 (2012).

[10] M. S. Hussein, M. P. Pato, and C. A. Bertulani, Phys. Rev. C 44, 2219 (1991). 